\title{
Synthesis and Biological Activity of the Lipophilic Derivatives of $\mathrm{N}$-Acetyl-1-thiomuramoyl-L-alanyl-D-isoglutamine ${ }^{\dagger}$
}

\author{
Akira Hasegawa, Shuichi Hara, Makoto Kiso \\ and Ichiro AzUMA* \\ Department of Agricultural Chemistry, Gifu University, \\ Gifu 501-11, Japan \\ * Institute of Immunological Science, Hokkaido University, \\ Sapporo 060, Japan
}

Received March 24, 1986

\begin{abstract}
A variety of the lipophilic derivatives at C-1 and C-6 in $N$-[2-O-(2-acetamido-2,3-dideoxy-1thio- $\beta$-D-glucopyranose-3-yl)-D-lactoyl]-L-alanyl-( $N^{1}$-fatty acyl)-D-isoglutamine methyl esters were synthesized from 2- $\mathrm{N}$-acetyl-1-S-acetyl-4,6-O-isopropylidene-1-thiomuramoyl-L-alanyl-D-isoglutamine methyl ester. Their immunoadjuvant activity in guinea-pigs, and the protective effect in mice infected with Escherichia coli (E-77156) were examined.
\end{abstract}

Recently, it has been shown that the introduction $^{1}{ }^{\sim 7}$ of fatty acyl groups as a lipophilic character at the restricted position of the sugar skeleton or dipeptide moiety in $\mathrm{N}$-acetylmuramoyl-L-alanyl-D-isoglutamine (MDP) and in its carbohydrate analogs that carry strong immunoadjuvant activity causes potent antitumor or anti-infection activity, based on the immune reaction, that is not found with MDP itself, and abolishes the pyrogenicity or necrotic inflammation that is a side effect with MDP.

In view of these facts, it seems important to elucidate the relationships between the position for introducing lipophilicity into the molecule and the chain length of the fatty acyl group as a lipophilic character, and their biological activities. We now describe the synthesis of lipophilic derivatives at both $\mathrm{C}-1$ or C-6 and the dipeptide moiety in $N$-acetyl-1thiomuramoyl-L-alanyl-D-isoglutamine methyl ester, ${ }^{8)}$ and their biological activities.

\section{RESULTS AND DISCUSSION}

Treatment of $\mathrm{N}$-[2-O-(2-acetamido-1-S- acetyl-2,3-dideoxy-4,6- $O$-isopropylidene- $\beta$ -D-glucopyranose-3-yl)-D-lactoyl]-L-alanylD-isoglutamine methyl ester ${ }^{8)}$ (1) with decanoyl chloride in pyridine and dichloromethane at $-5^{\circ} \mathrm{C}$ gave $N$-[2-O-(2-acetamido-1-Sacetyl-2,3-dideoxy-4,6- $O$-isopropylidene1-thio- $\beta$-D-glucopyranose-3-yl)-D-lactoyl]-Lalanyl-( $N^{1}$-decanoyl)-D-isoglutamine methyl ester (2). In the same way, condensation of 1 with tetradecanoyl or octadecanoyl chloride afforded the corresponding $N$-(fatty acyl) derivatives (3 and 4), respectively. $S$-Deacetylation of compounds $\mathbf{2} \sim \mathbf{4}$ with sodium methoxide in methanol, subsequent treatment with decanoic, tetradecanoic, or octadecanoic acid in dichloromethane, using dicyclohexylcarbodiimide (DCC) as the dehydrating agent, and $O$-deisopropylidenation with $80 \%$ aqueous acetic acid respectively yielded the corresponding $\quad N$-[2-O-(2-acetamide- $1-S$-acyl-2,3dideoxy-1-thio- $\beta$-D-glucopyranose-3-yl)-Dlactoyl]-L-alanyl-( $N^{1}$-acyl)-D-isoglutamine methyl esters $(\mathbf{5} \sim \mathbf{1 3})$.

On the other hand, $O$-deisopropylidenation of compounds $2 \sim \mathbf{4}$ by mildly acidic hydrolysis gave the corresponding crystalline $\mathrm{N}-[2-\mathrm{O}-(2-$ 

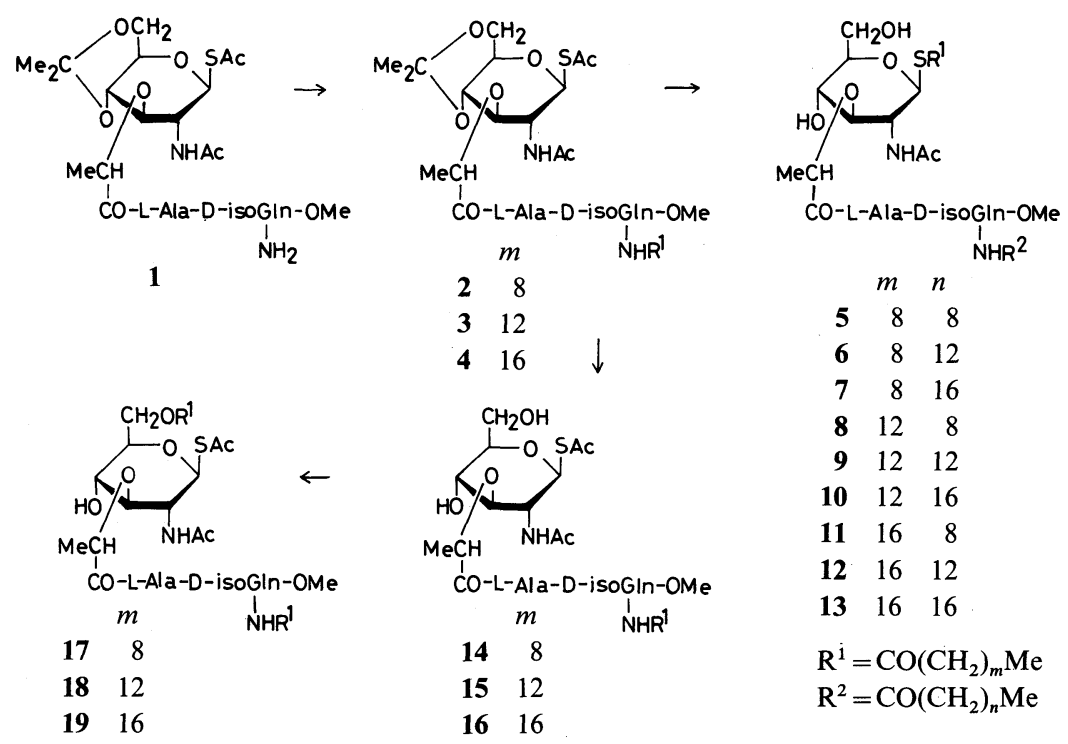

$\begin{array}{rrr}\mathbf{5} & 8 & 8 \\ \mathbf{6} & 8 & 12 \\ \mathbf{7} & 8 & 16 \\ \mathbf{8} & 12 & 8 \\ \mathbf{9} & 12 & 12 \\ \mathbf{1 0} & 12 & 16 \\ \mathbf{1 1} & 16 & 8 \\ \mathbf{1 2} & 16 & 12 \\ \mathbf{1 3} & 16 & 16 \\ \mathrm{R}^{\mathrm{i}}= & \mathrm{CO}\left(\mathrm{CH}_{2}\right)_{m} \mathrm{Me} \\ \mathrm{R}^{2}= & \mathrm{CO}\left(\mathrm{CH}_{2}\right)_{n} \mathrm{Me}\end{array}$

SCHEME

acetamido-1- $S$-acetyl-2,3-dideoxy- $\beta$-D-glucopyranose-3-yl)-D-lactoyl]-L-alanyl- $\left(N^{1}\right.$ acyl)-D-isoglutamine methyl esters $(\mathbf{4} \sim 16)$ in quantitative yields. When treated with decanoic, tetradecanoic, or octadecanoic acid in dry dichloromethane in the presence of DCC and 4-dimethylaminopyridine, compounds $14 \sim 16$ yielded the desired $N$-[2-O-(2-acetamido-6- $O$-acyl-1- $S$-acetyl-2,3-dideoxy- $\beta$ D-glucopyranose-3-yl)-D-lactoyl]-L-alanyl$\left(N^{1}\right.$-acyl)-D-isoglutamine methyl esters (17 19) in good yields.

The immunoadjuvant activity of the compounds thus obtained on the induction of the delayed type of hypersensitivity to $N$-acetyl-Ltyrosine-3-azobenzene- 4 '-arsonate (ABA- $N$ acetyltyrosine) in guinea-pigs was examined. ${ }^{9)}$

Compounds 5 13 had almost completely lost their activity, although the 1-S-acyl or 1-Sacyl-6- $O$-acyl-1-thio-MDP analogs showed strong immunoadjuvant activities, ${ }^{5 a, 8)}$ indicating that the introduction of a fatty acyl group at the isoglutamine moiety of $1-S$-acylMDP derivatives was unfavorable for the activity. However, compounds $14 \sim 16$ bearing an acyl group at the isoglutamine part exhibited strong adjuvant activity, almost comparable to that of MDP, indicating that the introduction of an acyl group at the isoglutamine moiety was not critical for the activity.

The protective activity in mice infected with Escherichia coli (E-77156) was examined. ${ }^{4)}$ Compounds 14 16, which showed potent adjuvant activity, provided efficient protection, but other compounds were inactive.

\section{EXPERIMENTAL}

Melting points $(\mathrm{mp})$ were determined with a Yanagimoto micro melting-point apparatus and are uncorrected. All evaporation was conducted in vacuo. Preparative chromatography was performed on silica gel (Waco Co., 200 mesh) with the solvent systems specified. Specific rotations were determined with a Union PM-201 polarimeter at $25^{\circ} \mathrm{C}$, and IR spectra were recorded with a Jasco A-100 spectrophotometer. NMR spectra were recorded with a Jeol JMN-GX 270 spectrometer.

$\mathrm{N}$-[2-O-(2-Acetamido-1-S-acetyl-2,3-dideoxy-4,6-Oisopropylidene-1-thio- $\beta$-D-glucopyranose-3-yl)-D-lactoyl]L-alanyl-( $N^{1}$-decanoyl)-D-isoglutamine methyl ester (2). To a solution of $\mathrm{N}$-[2-O-(2-acetamido-1-S-acetyl-4,6-O-isopropylidene-1-thio- $\beta$-D-glucopyranose-3-yl)-D-lactoyl]L-alanyl-D-isoglutamine methyl ester ${ }^{8)}(1,656 \mathrm{mg})$ in pyridine $(2 \mathrm{ml})$ and dichloromethane $(5 \mathrm{ml})$ was added, with stirring, a solution of decanoyl chloride $(1.1 \mathrm{~g})$ in dichloromethane $(15 \mathrm{ml})$ at $-5^{\circ} \mathrm{C}$. The mixture was stirred overnight at $0^{\circ} \mathrm{C}$, before methanol $(1 \mathrm{ml})$ was added, and the mixture was then evaporated to a syrup which was extracted with chloroform. The extract was washed with 
Table I. Adjuvant Activity of Some Lipophilic DeRIVATIVES OF 1-ThIOMURAMOYL-L-ALANYL-DISOGLUTAMINE ON THE INDUCTION OF DELAYED-TYPE HYPERSENSITIVITY TO ABA- $N$-ACETYLTYROSINE IN GUINEA-PIGS

\begin{tabular}{|c|c|c|}
\hline \multirow{2}{*}{ Compound $^{a}$} & \multicolumn{2}{|c|}{$\begin{array}{l}\text { Skin reaction with ABA-BSA }{ }^{b}(100 \mu \mathrm{g}) \\
(\text { diam. in } \mathrm{mm} \pm \text { S.E. })^{c}\end{array}$} \\
\hline & $24 \mathrm{hr}$ & $48 \mathrm{hr}$ \\
\hline 5 & $(8.8 \pm 0.6)$ & 0 \\
\hline 6 & $(8.0 \pm 0.8)$ & 0 \\
\hline 7 & $(14.5 \pm 1.6)$ & $(11.0 \pm 2.6)$ \\
\hline 8 & $(10.5 \pm 0.6)$ & 0 \\
\hline 9 & 0 & 0 \\
\hline 10 & 0 & 0 \\
\hline 11 & 0 & 0 \\
\hline 12 & 0 & 0 \\
\hline 13 & 0 & 0 \\
\hline 14 & $16.7 \pm 1.8$ & $13.8 \pm 1.4$ \\
\hline 15 & $15.5 \pm 2.3$ & $13.8 \pm 1.0$ \\
\hline 16 & $16.1 \pm 1.5$ & $16.9 \pm 0.6$ \\
\hline 17 & 0 & 0 \\
\hline 18 & $(18.1 \pm 2.0)$ & $(17.5 \pm 1.9)$ \\
\hline 19 & $(17.8 \pm 2.7)$ & $(14.8 \pm 1.2)$ \\
\hline MDP & $22.5 \pm 0.4$ & $16.3 \pm 0.6$ \\
\hline Control $^{d}$ & 0 & 0 \\
\hline
\end{tabular}

a Dose: $10 \mu \mathrm{g}$.

b Azobenzenearsonate- $N$-acetyl-L-tyrosine-bovine serum albumin.

c The data indicate the average diameter \pm standard error (S.E.) of skin reaction (induration) in four guinea-pigs; the values in parentheses indicate the size of erythema.

d ABA- $N$-Acetyltyrosine in Freund's incomplete adjuvant.

water, dried (sodium sulfate) and evaporated to a syrup, which was chromatographed on a column of silica gel (60 g) with (a) $150: 1$, (b) $100: 1$, and (c) $70: 1$ chloroform-methanol. Eluant (c) gave compound $2(390 \mathrm{mg}$, $47 \%)$ as a syrup; $[\alpha]_{\mathrm{D}}+3.9^{\circ}(c=0.9$, chloroform); IR $v_{\max }^{\text {film }} \mathrm{cm}^{-1}: 3280(\mathrm{NH}), 2930$ and $2850(\mathrm{Me}$, methylene), 1740 and 1260 (ester), 1700 ( $S$-acetyl), 1660 and 1550 (amide), and $860\left(\mathrm{Me}_{2} \mathrm{C}\right)$; NMR $\left(\mathrm{CDCl}_{3}\right): \delta 0.88(\mathrm{t}, 3 \mathrm{H}$, $\left.J_{\mathrm{Me}, \mathrm{CH}_{2}} 6.6 \mathrm{~Hz}, \mathrm{MeCH}_{2}\right), 1.25\left(\mathrm{~s}, 14 \mathrm{H}, 7 \mathrm{CH}_{2}\right), 1.36(\mathrm{~d}, 3 \mathrm{H}$, $\left.J_{\mathrm{Me}, \mathrm{CH}} 6.6 \mathrm{~Hz}, \mathrm{MeCH}\right), 1.41\left(\mathrm{~d}, 3 \mathrm{H}, J_{\mathrm{Me}, \mathrm{CH}} 7.7 \mathrm{~Hz}, \mathrm{MeCH}\right)$, $1.40,1.50$ (2s, 6H, Me $\left.{ }_{2} \mathrm{C}\right), 1.94$ (s, 3H, AcN), 2.37 (s, 3H, $\mathrm{AcS}), 3.68(\mathrm{~s}, 3 \mathrm{H}, \mathrm{MeO})$, and $5.27\left(\mathrm{~d}, 1 \mathrm{H}, J_{1,2} 10.6 \mathrm{~Hz}\right.$, H-1).

Anal. Found: C, 55.21; H, 7.89; N, 3.36. Calcd. for $\mathrm{C}_{35} \mathrm{H}_{58} \mathrm{~N}_{4} \mathrm{O}_{12} \mathrm{~S}$ : C, 55.39; $\mathrm{H}, 7.70 ; \mathrm{N}, 7.38 \%$.

Other $N$-[2-O-(2-acetamido-1-S-acetyl-2,3-dideoxy-4,6$O$-isopropylidene-1-thio- $\beta-D-$ glucopyranose-3-yl)-D-
lactoyl]-L-alanyl-( $N^{1}$-acyl)-D-isoglutamine methyl esters (3 and 4). These were prepared from compound 1 by $N$ acylation with tetradecanoyl or octadecanoyl chloride, according to the procedure described for compound $\mathbf{2}$.

Compound 3 was obtained as a syrup $(48 \%) ;[\alpha]_{\mathrm{D}}+$ $2.1^{\circ}\left(c=0.9\right.$, chloroform); IR $v_{\max }^{\mathrm{film}} \mathrm{cm}^{-1}: 3280(\mathrm{NH}), 2930$ and 2850 (Me, methylene), 1740 and 1260 (ester), 1700 ( $S$ acetyl), 1660 and 1530 (amide), and $860\left(\mathrm{Me}_{2} \mathrm{C}\right)$; NMR $\left(\mathrm{CDCl}_{3}\right): \delta 0.87\left(\mathrm{t}, 3 \mathrm{H}, J_{\mathrm{Me}, \mathrm{CH}_{2}} 6.4 \mathrm{~Hz}, \mathrm{MeCH}_{2}\right), 1.26(\mathrm{~s}$, $\left.22 \mathrm{H}, 11 \mathrm{CH}_{2}\right), 1.38\left(\mathrm{~d}, 3 \mathrm{H}, J_{\mathrm{Me}, \mathrm{CH}} 6.6 \mathrm{~Hz}, \mathrm{MeCH}\right), 1.39$, $1.50\left(2 \mathrm{~s}, 6 \mathrm{H}, \mathrm{Me}_{2} \mathrm{C}\right), 1.43\left(\mathrm{~d}, 3 \mathrm{H}, J_{\mathrm{Me}, \mathrm{CH}} 7.3 \mathrm{~Hz}, \mathrm{MeCH}\right)$, 1.95 (s, 3H, AcN), 2.37 (s, 3H, AcS), 3.68 (s, 3H, MeO), and $5.27\left(\mathrm{~d}, 1 \mathrm{H}, J_{1,2} 10.6 \mathrm{~Hz}, \mathrm{H}-1\right)$.

Anal. Found: C, 58.82; H, 8.81; N, 7.15. Calcd. for $\mathrm{C}_{39} \mathrm{H}_{66} \mathrm{~N}_{4} \mathrm{O}_{12} \mathrm{~S}: \mathrm{C}, 59.83 ; \mathrm{H}, 8.50 ; \mathrm{N}, 7.16 \%$.

Compound 4 was obtained as a syrup $(45 \%) ;[\alpha]_{\mathrm{D}}+$ $2.5^{\circ}\left(c=0.4\right.$, chloroform); IR $v_{\max }^{\text {film }} \mathrm{cm}^{-1}: 3280(\mathrm{NH}), 2930$ and 2850 (Me, methylene), 1740 and 1260 (ester), 1700 (Sacetyl), 1660 and 1530 (amide), and $855\left(\mathrm{Me}_{2} \mathrm{C}\right)$; $\mathrm{NMR}$ $\left(\mathrm{CDCl}_{3}\right): \delta 0.88\left(\mathrm{t}, 3 \mathrm{H}, J_{\mathrm{Me}, \mathrm{CH}_{2}} 6.6 \mathrm{~Hz}, \mathrm{MeCH}_{2}\right), 1.26(\mathrm{~s}$, $\left.30 \mathrm{H}, 15 \mathrm{CH}_{2}\right), 1.36,1.38\left(2 \mathrm{~d}, 6 \mathrm{H}, J_{\mathrm{Me}, \mathrm{CH}} 7.6 \mathrm{~Hz}, 2 \mathrm{MeCH}\right)$, $1.40,1.50$ (2s, 6H, $\left.\mathrm{Me}_{2} \mathrm{C}\right), 1.96$ (s, 3H, AcN), 2.37 (s, 3H, AcS), 3.68 (s, 3H, MeO), and $5.27\left(\mathrm{~d}, 1 \mathrm{H}, J_{1,2} 10.6 \mathrm{~Hz}, \mathrm{H}-\right.$ 1).

Anal. Found: C, 59.18; H, 8.60; N, 6.37. Calcd. for $\mathrm{C}_{43} \mathrm{H}_{74} \mathrm{~N}_{4} \mathrm{O}_{12} \mathrm{~S}$ : C, 59.29; H, 8.56; N, 6.43\%.

$N$-[2-O-(2-Acetamido-1-S-decanoyl-2,3-dideoxy-1thio- $\beta$-D-glucopyranose-3-yl)-D-lactoyl]-L-alanyl- $\left(N^{1}\right.$ decanoyl)-D-isoglutamine methyl ester (5). To a solution of $2(44 \mathrm{mg})$ in methanol $(2 \mathrm{ml})$ was added sodium methoxide $(10 \mathrm{mg})$. The mixture was kept for $20 \mathrm{~min}$ at $0^{\circ} \mathrm{C}$, treated with Amberlite IR-120 $\left(\mathrm{H}^{+}\right)$resin to remove the base, and evaporated. The residue was dissolved in dry dichloromethane $(2 \mathrm{ml})$, and decanoic acid $(15 \mathrm{mg})$ and dicyclohexylcarbodiimide (DCC) $(23 \mathrm{mg}$ ) were added to the solution. The mixture was stirred for $2 \mathrm{hr}$ at room temperature, the course of the reaction being monitored by TLC. After evaporating the solvent, the residue was chromatographed on a column of silica gel $(5 \mathrm{~g})$ with $100: 1$ and $70: 1$ chloroform-methanol. The latter eluate gave the 1-S(fatty acyl) derivative, which was dissolved in $80 \%$ aqueous acetic acid $(5 \mathrm{ml})$, heated for $6 \mathrm{hr}$ at $45^{\circ} \mathrm{C}$, and then evaporated. Crystallization from ether-hexane gave compound $5(25 \mathrm{mg}, 52 \%) ; \mathrm{mp} 210^{\circ} \mathrm{C},[\alpha]_{\mathrm{D}}+4.1^{\circ} \quad(c=0.2$, methanol); IR $v_{\max }^{\mathrm{KBr}} \mathrm{cm}^{-1}: 3280(\mathrm{OH}, \mathrm{NH}), 2940$ and 2850 (Me, methylene), 1740 and 1240 (ester), 1700 ( $S$-acyl), and 1650 and 1550 (amide); NMR (1:1 $\left.\mathrm{CDCl}_{3}-\mathrm{CD}_{3} \mathrm{OD}\right)$ : $\delta 0.88\left(2 \mathrm{t}, 6 \mathrm{H}, J_{\mathrm{Me}, \mathrm{CH}_{2}} 6.6 \mathrm{~Hz}, 2 \mathrm{MeCH}_{2}\right), 1.26(\mathrm{~s}, 28 \mathrm{H}$, $\left.14 \mathrm{CH}_{2}\right), 1.38\left(\mathrm{~d}, 3 \mathrm{H}, J_{\mathrm{Me}, \mathrm{CH}} 6.6 \mathrm{~Hz}, \mathrm{MeCH}\right), 1.40(\mathrm{~d}, 3 \mathrm{H}$, $\left.J_{\mathrm{Me}, \mathrm{CH}} 7.0 \mathrm{~Hz}, \mathrm{MeCH}\right), 1.91$ (s, 3H, AcN), 3.69 (s, 3H, $\mathrm{MeO})$, and $5.12\left(\mathrm{~d}, 1 \mathrm{H}, J_{1,2} 10.6 \mathrm{~Hz}, \mathrm{H}-1\right)$.

Anal. Found: C, 57.77; H, 8.53; N, 6.70. Calcd. for $\mathrm{C}_{40} \mathrm{H}_{70} \mathrm{~N}_{4} \mathrm{O}_{12} \mathrm{~S}: \mathrm{C}, 57.81 ; \mathrm{H}, 8.49 ; \mathrm{N}, 6.74 \%$.

Other $N$-[2-O-(2-acetamido-1-S-acyl-2,3-dideoxy-1thio- $\beta$-D-glucopyranose-3-yl)-D-lactoyl]-L-alanyl- $\left(N^{1}\right.$. 
acyl)-D-isoglutamine methyl esters $(6 \sim 13)$. These were respectively prepared from compounds $2 \sim 4$, according to the method described for $\mathbf{5}$, and all of the compounds thus obtained showed similar IR and NMR spectra, which were consistent with the structures assigned.

Compound 6: $51 \%$ yield, $\mathrm{mp} 158 \sim 159^{\circ} \mathrm{C},[\alpha]_{\mathrm{D}}+13^{\circ}$ ( $c=0.4,1: 1$ chloroform-methanol).

Anal. Found: C, 59.55; H, 8.91; N, 6.26. Calcd. for $\mathrm{C}_{44} \mathrm{H}_{78} \mathrm{~N}_{4} \mathrm{O}_{12} \mathrm{~S}: \mathrm{C}, 59.57 ; \mathrm{H}, 8.86 ; \mathrm{N}, 6.32 \%$.

Compound 7: $49 \%$ yield, mp $147 \sim 148^{\circ} \mathrm{C},[\alpha]_{\mathrm{D}}+26.5^{\circ}$ ( $c=0.6,1: 1$ chloroform-methanol).

Anal. Found: C, 60.98; H, 9.28; N, 5.91. Calcd. for $\mathrm{C}_{48} \mathrm{H}_{86} \mathrm{~N}_{4} \mathrm{O}_{12} \mathrm{~S}: \mathrm{C}, 61.12 ; \mathrm{H}, 9.19 ; \mathrm{N}, 5.94 \%$.

Compound 8: $58 \%$ yield, mp $179^{\circ} \mathrm{C},[\alpha]_{\mathrm{D}}+29.5^{\circ}(c=0.7$, $1: 1$ chloroform-methanol).

Anal. Found: C, 59.55; H, 8.79; N, 6.28. Calcd. for $\mathrm{C}_{44} \mathrm{H}_{78} \mathrm{~N}_{4} \mathrm{O}_{12} \mathrm{~S}: \mathrm{C}, 59.57 ; \mathrm{H}, 8.86 ; \mathrm{N}, 6.32 \%$.

Compound 9: $61 \%$ yield, mp $162 \sim 163^{\circ} \mathrm{C},[\alpha]_{\mathrm{D}}+7.5^{\circ}$ ( $c=0.5,6: 1$ chloroform-methanol). .

Anal. Found: C, 61.12; H, 9.33; N, 5.81. Calcd. for $\mathrm{C}_{48} \mathrm{H}_{86} \mathrm{~N}_{4} \mathrm{O}_{12} \mathrm{~S}: \mathrm{C}, 61.12 ; \mathrm{H}, 9.19 ; \mathrm{N}, 5.94 \%$.

Compound 10: $68 \%$ yield, mp $158 \sim 159^{\circ} \mathrm{C},[\alpha]_{\mathrm{D}}+14^{\circ}$ ( $c=0.5,6: 1$ chloroform-methanol).

Anal. Found: C, 62.38; H, 9.39; N, 5.55. Calcd. for $\mathrm{C}_{52} \mathrm{H}_{94} \mathrm{~N}_{4} \mathrm{O}_{12} \mathrm{~S}$ : C, 62.49; H, 9.48; N, 5.61\%.

Compound 11: $61 \%$ yield. $\mathrm{mp} 166^{\circ} \mathrm{C},[\alpha]_{\mathrm{D}}+37.5^{\circ}(c=$ 1.0, $1: 1$ chloroform-methanol).

Anal. Found: C, 61.06; H, 9.25; N, 5.88. Calcd. for $\mathrm{C}_{48} \mathrm{H}_{86} \mathrm{~N}_{4} \mathrm{O}_{12} \mathrm{~S}: \mathrm{C}, 61.12 ; \mathrm{H}, 9.19 ; \mathrm{N}, 5.94 \%$.

Compound 12: $76 \%$ yield, mp $152 \sim 153^{\circ} \mathrm{C},[\alpha]_{\mathrm{D}}+36.5^{\circ}$ ( $c=1: 1$ chloroform-methanol).

Anal. Found: C, 62.39; H, 9.61; N, 5.65. Calcd. for $\mathrm{C}_{52} \mathrm{H}_{94} \mathrm{~N}_{4} \mathrm{O}_{12} \mathrm{~S}$ : C, 62.49; H, 9.48; N, 5.61\%.

Compound 13: $49 \%$ yield, mp $151 \sim 152^{\circ} \mathrm{C},[\alpha]_{\mathrm{D}}+$ $27.6^{\circ}$ ( $c=0.6,1: 2$ chloroform-methanol).

Anal. Found: C, 63.70; H, 9.93; N, 5.28. Calcd. for $\mathrm{C}_{56} \mathrm{H}_{102} \mathrm{~N}_{4} \mathrm{O}_{12} \mathrm{~S}: \mathrm{C}, 63.72 ; \mathrm{H}, 9.74 ; \mathrm{N}, 5.31 \%$.

$N$-[2-O-(2-Acetamido-1-S-acetyl-2,3-dideoxy-1-thio$\beta$-D-glucopyranose-3-yl)-D-lactoyl]-L-alanyl- $\left(N^{1}\right.$-decanoyl)-D-isoglutamine methyl ester (14). A solution of 2 $(120 \mathrm{mg})$ in $80 \%$ aqueous acetic acid $(4 \mathrm{ml})$ was heated for $6 \mathrm{hr}$ at $45^{\circ} \mathrm{C}$, and evaporated to a crystalline mass. Recrystallization from ether-hexane gave $14(115 \mathrm{mg}$, quantitative); mp $175 \sim 176^{\circ} \mathrm{C},[\alpha]_{\mathrm{D}}+29^{\circ}(c=1.1$, methanol); IR $v_{\max }^{\mathrm{KBr}} \mathrm{cm}^{-1}: 3280(\mathrm{OH}, \mathrm{NH}), 2940$ and $2850(\mathrm{Me}$, methylene), 1750 and 1270 (ester), 1700 ( $S$-acetyl), and 1650 and 1540 (amide).

Anal. Found: C, 53.51; H, 7.66; N, 7.75. Calcd. for $\mathrm{C}_{32} \mathrm{H}_{54} \mathrm{~N}_{4} \mathrm{O}_{12} \mathrm{~S}: \mathrm{C}, 53.47 ; \mathrm{H}, 7.57 ; \mathrm{N}, 7.79 \%$.

Other $\quad \mathrm{N}$-[2-O-(2-acetamido-1-S-acetyl-2,3-dideoxy-1thio- $\beta$-D-glucopyranose-3-yl)-D-lactoyl $]$ - L-alanyl- $\left(N^{1}\right.$ acyl)-D-isoglutamine methyl esters (15 and 16). These were respectively prepared from $\mathbf{3}$ and $\mathbf{4}$ by hydrolysis, as described for compound 14.

Compound 15: quantitative yield, $\mathrm{mp} 175 \sim 176^{\circ} \mathrm{C}$,
$[\alpha]_{\mathrm{D}}+27^{\circ}(c=1.1$, methanol).

Anal. Found: C, 55.86; H, 8.13; N, 7.15. Calcd. for $\mathrm{C}_{36} \mathrm{H}_{62} \mathrm{~N}_{4} \mathrm{O}_{12} \mathrm{~S}$ : C, 55.79; $\mathrm{H}, 8.06 ; \mathrm{N}, 7.23 \%$.

Compound 16: quantitative yield, $\mathrm{mp} 160 \sim 161^{\circ} \mathrm{C}$, $[\alpha]_{\mathrm{D}}+22.5^{\circ}(c=0.9$, methanol).

Anal. Found: C, 57.71; H, 8.53; N, 6.73. Calcd. for $\mathrm{C}_{40} \mathrm{H}_{70} \mathrm{~N}_{4} \mathrm{O}_{12} \mathrm{~S}: \mathrm{C}, 57.81 ; \mathrm{H}, 8.49 ; \mathrm{N}, 6.73 \%$.

$N$-[2-O-(2-Acetamido-1-S-acetyl-6-O-decanoyl-2,3dideoxy-1-thio- $\beta$-D-glucopyranose-3-yl)-D-lactoyl $]-L$ alanyl-( $N^{1}$-decanoyl)-D-isoglutamine methyl ester (17). To a solution of $14(50 \mathrm{mg})$ in dry dichloromethane $(3 \mathrm{ml})$ were added, with stirring, decanoic acid $(13.5 \mathrm{mg})$, DCC $(29.5 \mathrm{mg})$ and 4-dimethylaminopyridine $(4.3 \mathrm{mg})$, the mixture being stirred for $2 \mathrm{hr}$ at room temperature, and then evaporated. The residue was chromatographed on a column of silica gel $(10 \mathrm{~g})$ with (a) $100: 1$, (b) $70: 1$, and (c) $50: 1$ chloroform-methanol. Eluant (c) afforded 17 $\left(30 \mathrm{mg}, 48 \%\right.$ ) as crystals, mp $145 \sim 146^{\circ} \mathrm{C},[\alpha]_{\mathrm{D}}-12^{\circ}(c=$ 1.0 , chloroform); IR $v_{\max }^{\mathrm{KBr}} \mathrm{cm}^{-1}: 3280(\mathrm{OH}, \mathrm{NH}), 2930$ and 2850 (Me, methylene), 1730 and 1260 (ester), 1700 ( $S$ acetyl), and 1640 and 1535 (amide); NMR ( $\left.\mathrm{CD}_{3} \mathrm{OD}\right)$ : $\delta 0.85 \sim 0.92\left(\mathrm{~m}, 6 \mathrm{H}, 2 \mathrm{MeCH}_{2}\right), 1.26\left(\mathrm{~s}, 28 \mathrm{H}, 14 \mathrm{CH}_{2}\right)$, $1.36,1.41\left(2 \mathrm{~d}, 6 \mathrm{H}, J_{\mathrm{Me}, \mathrm{CH}} 7.0 \mathrm{~Hz}, 2 \mathrm{MeCH}\right), 1.96(\mathrm{~s}, 3 \mathrm{H}$, $\mathrm{AcN}), 2.35(\mathrm{~s}, 3 \mathrm{H}, \mathrm{AcS})$, and $5.15\left(\mathrm{~d}, 1 \mathrm{H}, J_{1,2} 10.6 \mathrm{~Hz}, \mathrm{H}-\right.$ 1).

Anal. Found: C, 57.66; H, 8.35; N, 6.41. Calcd. for $\mathrm{C}_{42} \mathrm{H}_{72} \mathrm{~N}_{4} \mathrm{O}_{13} \mathrm{~S}$ : C, 57.78; H, 8.31; N, 6.42\%.

Other N[2-O-(2-acetamido-1-S-acetyl-6-O-acyl-2,3-dideoxy-1-thio- $\beta$-D-glucopyranose-3-yl)-D-lactoyl]-Lalanyl- $\left(N^{1}-a c y l\right)$-D-isoglutamine methyl esters (18 and 19). These were prepared by 6-O-acylation of 15 and 16, according to the procedure described for 17.

Compound 18: $63 \%$ yield, mp $128 \sim 129^{\circ} \mathrm{C},[\alpha]_{\mathrm{D}}-4.7^{\circ}$ ( $c=0.86$, chloroform); IR $v_{\max }^{\mathrm{KBr}} \mathrm{cm}^{-1}: 3280(\mathrm{OH}, \mathrm{NH})$, 2930 and 2850 (Me, methylene), 1740, 1730 and 1260 (ester), 1700 ( $S$-acetyl), and 1640 and 1530 (amide); NMR $\left(\mathrm{CDCl}_{3}\right): \delta 0.88\left(\mathrm{t}, 6 \mathrm{H}, J_{\mathrm{Me}, \mathrm{CH}_{2}} 6.8 \mathrm{~Hz}, 2 \mathrm{MeCH}_{2}\right), 1.26(\mathrm{~s}$, $\left.44 \mathrm{H}, 22 \mathrm{CH}_{2}\right), 1.35,1.41\left(2 \mathrm{~d}, 6 \mathrm{H}, J_{\mathrm{Me}, \mathrm{CH}} 7.0 \mathrm{~Hz}, 2 \mathrm{MeCH}\right)$, 1.97 (s, 3H, AcN), 2.36 (s, 3H, AcS), 3.69 (s, 3H, MeO), and $5.15\left(\mathrm{~d}, 1 \mathrm{H}, J_{1,2} 10.6 \mathrm{~Hz}, \mathrm{H}-1\right)$.

Anal. Found: C, 60.77; H, 9.23; N, 5.58. Calcd. for $\mathrm{C}_{50} \mathrm{H}_{88} \mathrm{~N}_{4} \mathrm{O}_{13} \mathrm{~S}$ : C, 60.95; H, 9.00; N, 5.69\%.

Compound 19: $69 \%$ yield, mp $117 \sim 118^{\circ} \mathrm{C},[\alpha]_{\mathrm{D}}-7.3^{\circ}$ $\left(c=0.55\right.$, chloroform); IR $v_{\max }^{\mathrm{KBr}} \mathrm{cm}^{-1}: 3280(\mathrm{OH}, \mathrm{NH})$, 2930 and 2850 (Me, methylene), 1740 and 1260 (ester), 1700 ( $S$-acetyl), and 1660 and 1540 (amide); NMR $\left(\mathrm{CDCl}_{3}\right): \delta 0.88\left(\mathrm{t}, 6 \mathrm{H}, J_{\mathrm{Me}, \mathrm{CH}_{2}}, 6.6 \mathrm{~Hz}, 2 \mathrm{MeCH}_{2}\right), 1.25(\mathrm{~s}$, $\left.60 \mathrm{H}, 30 \mathrm{CH}_{2}\right), 1.36,1.41\left(2 \mathrm{~d}, 6 \mathrm{H}, J_{\mathrm{Me}, \mathrm{CH}} 7.0 \mathrm{~Hz}, 2 \mathrm{MeCH}\right)$, 1.98 (s, 3H, AcN), 2.36 (s, 3H, AcS), 3.69 (s, 3H, MeO), and $5.15\left(\mathrm{~d}, 1 \mathrm{H}, J_{1,2} 10.7 \mathrm{~Hz}, \mathrm{H}-1\right)$.

Anal. Found: C, 63.52; H, 9.48; N, 5.05. Calcd. for $\mathrm{C}_{58} \mathrm{H}_{104} \mathrm{~N}_{4} \mathrm{O}_{13} \mathrm{~S}: \mathrm{C}, 63.47 ; \mathrm{H}, 9.55 ; \mathrm{N}, 5.10 \%$.

Acknowledgments. This work was supported in part by a Grant-in-Aid for Special Project Research from the 
Ministry of Education, Science and Culture of Japan, and by a Naito Research Grant.

\section{REFERENCES}

1) A. Hasegawa, K. Kigawa, M. Kiso and I. Azuma, Agric. Biol. Chem., 50, 2091 (1986).

2) S. Kusumoto, M. Inage, T. Shiba, I. Azuma and Y. Yamamura, Tetrahedron Lett., 1978, 4899.

3) S. Kobayashi, T. Fukuta, I. Imada, M. Fujino, I. Azuma and Y. Yamamura, Chem. Pharm. Bull., 27, 3193 (1979).

4) K. Matsumoto, T. Otani, T. Une, Y. Osada, H. Ogawa and I. Azuma, Infect. Immun., 39, 1029 (1983).
5) (a) A. Hasegawa, Y. Hioki, M. Kiso, H. Okumura and I. Azuma, Carbohydr. Res., 123, 183 (1983); (b) H. Okumura, K. Kamisango, I. Saiki, Y. Tanio, I. Azuma, M. Kiso and A. Hasegawa, Agric. Biol. Chem., 46, 507 (1982).

6) P. Lefrancier, M. Petiton, M. Level, M. Derrien, J. Choay and E. Lederer, J. Peptide Protein Res., 14, 437 (1979).

7) J. Schriot, E. Galligioni and J. Fidler, Biol. Cell., 47, 87 (1983).

8) A. Hasegawa, Y. Hioki, M. Kiso, H. Okumura and I. Azuma, J. Carbohydr. Chem., 1, 317 (1982 83).

9) I. Azuma, H. Okumura, I. Saiki, Y. Tanio, M. Kiso, A. Hasegawa and Y. Yamamura, Infect. Immun., 32, 1305 (1981). 